\title{
Intoxicação Aguda por Nitrogênio através de Inalação em Paciente com Insuficiência Respiratória e Coma. Relato de Caso*
}

\author{
Acute Nitrogen Intoxication by Patient Inhalation with \\ Breathing Insufficiency and Coma. Case Report.
}

\author{
Pedro Henrique Masjuan Torrecillas ${ }^{1}$, Maria Aparecida Nogueira de Barros ${ }^{1}$, \\ Valeria Lima Cruz², Audineia Aparecida Martins ${ }^{3}$, João Antônio Aidar Coelho ${ }^{4}$
}

\section{RESUMO}

JUSTIFICATIVA E OBJETIVOS: Apresentação de um caso único não encontrado na literatura nacional. $\mathrm{O}$ objetivo deste relato foi apresentar um caso de grave intoxicação por nitrogênio líquido (NO), com edema agudo de pulmão e encefalopatia isquêmica, em que se obteve bom desfecho, provavelmente, pela rapidez no atendimento e na administração adequada da terapêutica.

RELATO DO CASO: Paciente do sexo masculino, 26 anos, encontrado desacordado havia aproximadamente uma hora, em sala fechada onde havia uma máquina utilizada para manutenção do gelo de pista de patinação. Constatou-se aspiração de conteúdo gástrico e edema das vias respiratórias altas. A intubação na emergência foi difícil pelo intenso edema de laringe. O paciente desenvolveu edema agudo de pulmão e sinais de edema encefálico por encefalopatia anóxica. Evoluiu com melhora pulmonar lenta com ventilação protetora para síndrome da angústia respiratória aguda (volume corrente de $5 \mathrm{~mL} / \mathrm{kg}$, PEEP de $15 \mathrm{cmH}_{2} \mathrm{O}$ ) e corticoterapia com hidrocortisona (200 mg) a cada seis horas para

1. Diarista da UTI do Hospital Regional do Vale do Paraíba

2. Coordenadora da UTI do Hospital Regional do Vale do Paraíba

3. Diretora Técnica e Clínica do Hospital Regional do Vale do Paraíba.

4. Diretor Executivo do Hospital Regional do Vale do Paraíba.

*Recebido do Hospital Regional Vale do Paraíba, Taubaté, SP

Apresentado em 21 de novembro de 2005

Aceito para publicação 14 de março de 2006

Endereço para correspondência:

Dr. Pedro Henrique Masjuan Torrecillas

Hospital Regional do Vale do Paraíba,

Av. Tiradentes 280

12030-180 Taubaté, SP

Fone: 12 3634-2000

E-mail: pedrohm@uol.com.br

(C)Associação de Medicina Intensiva Brasileira, 2006 tratamento de broncoespasmo.

CONCLUSÕES: Trata-se do primeiro caso publicado em nosso meio de intoxicação por nitrogênio. Na literatura internacional encontram-se várias citações de situações semelhantes ao deste caso pelo fato de existir varias pistas de gelo para prática de esportes como por exemplo o hockey. Há relatos de exacerbações de quadros de broncoespasmo nas pessoas que assistem ao jogo, apresentando-se de forma tardia, por vezes até sete a dez horas após a exposição aos vapores que exalam destas pistas que são ricas em nitrogênio.

Unitermos: broncoespasmo, edema de laringe, Lesão pulmonar aguda, nitrogênio líquido

\section{SUMMARY}

BACKGROUND AND OBJECTIVES: To present the first case reported in the Brazilian literature of liquid nitrogen intoxication. The objective of this report was to present a case of severe intoxication by liquid nitrogen, with acute lung edema and ischemic encephalopathy, which a good outcome, due to a fast diligence and a proper therapy administration.

CASE REPORT: Male patient, 26 years, unconscious for one hour, in a close room with a machine to keep frozen the ice-roller ring. There was evidence of gastric aspiration and laryngeal edema. The intubation was difficult. The patient developed acute lung edema and brain edema due to hypoxia. There was a slow lung resolution with a protective ventilatory strategy for ARDS (VT $5 \mathrm{~mL}$ / $\mathrm{kg}$, PEEP de $15 \mathrm{cmH}_{2} \mathrm{O}$ ) and corticotherapy with hydrocortisone $(200 \mathrm{mg})$ every $6 \mathrm{~h}$ to treat bronchospasm.

CONCLUSIONS: This the first case published in Brazil of nitrogenintoxication. Intheinternationalliteraturethereare several reports of similar cases occurred in gymnasiums for hockey game. There are reports of bronchospasm 
exacerbations in people after the matches, even up to $10 \mathrm{~h}$ after exposal to the gases riches in nitrogen.

Key Words: acute lung damage, bronchospam, laryngeal edema, Liquid nitrogen

\section{INTRODUÇÃO}

O óxido nítrico (NO) é um gás que está presente no ar ambiente e como produto de combustão de veículos automotores, sendo utilizado na forma líquida para processos de resfriamento'.

A exposição humana ao NO leva à oxidação transformando-se em dióxido de nitrogênio que funciona como um segundo mensageiro intracelular modulando a ação de uma série de enzimas ${ }^{1}$. O óxido nítrico ativa a enzima guanilil-ciclase que incrementa a produção de cGMP. Como conseqüência pode-se ter vasodilatação da circulação pulmonar com aumento da permeabilidade capilar e edema, com inundação alveolar caracterizando edema agudo pulmonar ${ }^{1}$. A inalação de altas concentrações do gás leva a lesões das mucosas nasal e laringo-brônquica com denudação da mucosa e grande edema, dificultando, por vezes, a intubação. Quando se tem alta concentração de NO associada a um ambiente fechado pode-se ter uma competição com o oxigênio do ar o que acarreta uma síndrome de hipóxia nas pessoas que estão expostas a este ambiente com sintomas de encefalopatia isquêmica ${ }^{2,3}$.

O quadro clínico varia conforme a quantidade inalada assim como a velocidade em que o gás é absorvido. Em ambientes fechados onde se utiliza o NO para manutenção de pistas de gelo há relatos de tosse, broncoespasmo e sintomas semelhantes à rinite que surgem entre 5 e 72 horas após a exposição, sendo que em pessoas com perfil asmático ou DOPC pode ocorrer crises mais graves levando estes pacientes ao serviço de emergência num período mais curto ${ }^{4-7}$.

Quando a intoxicação é mais intensa, têm-se sintomas de edema agudo de pulmão com instabilidade hemodinâmica alem de quadro neurológico que varia de confusão mental até coma em decorrência da encefalopatia anóxica'.

O objetivo deste relato foi apresentar um caso de grave intoxicação por nitrogênio líquido, com edema agudo de pulmão e encefalopatia isquêmica, em que se obteve bom desfecho, provavelmente, pela rapidez no atendimento e na administração adequada da terapêutica.

\section{RELATO DO CASO}

Paciente do sexo masculino, 26 anos, foi encontrado em estado de coma em sala fechada com vazamento de nitrogênio proveniente de maquina utilizada para resfriamento de pista de gelo, com aproximadamente uma hora de exposição ao gás. Foi imediatamente transferido para o pronto socorro, onde foi intubado em decorrência de insuficiência respiratória. Encontrava-se confuso, desorientado, com pulso fino e forte suspeita de aspiração de conteúdo gástrico.

Deu entrada na UTI do Hospital Regional de Taubaté com escala de coma de Glasgow 8, edema de face, língua e lábios com coloração rosada alem de petéquias na região de conjuntivas. Pressão arterial $100 \mathrm{x}$ $50 \mathrm{mmHg}$, ritmo cardíaco e sinais evidentes de edema agudo de pulmão, com crepitação em ambos os hemitórax e presença de secreção rósea pelo tubo de intubação orotraqueal. Havia sinais claros de aspiração de conteúdo gástrico para o pulmão.

Recebeu sedação contínua, ventilação mecânica com PEEP ajustada para 10, $\mathrm{F}_{1} \mathrm{O}_{2} 90 \%$ mantendo a $\mathrm{PaO}_{2}$ arterial de 150 e foram administrados antibiótico e corticosteróide.

Foi feita radiografia de tórax e tomografia do crânio. As figuras 1, 2 e 3, mostram derrame pleural bilateral com infiltrado pneumônico à direita alem de sinais sugestivos de swelling encefálico.

Permaneceu em ventilação mecânica por quatro dias com melhora progressiva dos parâmetros ventilatórios sendo extubado no $5^{\circ}$ dia de UTI tendo alta sem seqüela neurológica. Tomografia de controle no segundo dia de internação já mostrava diminuição dos sinais de swelling encefálico.

Durante a internação, o paciente permaneceu com estabilidade hemodinâmica, não sendo necessário utilização de fármacos vasoativos.

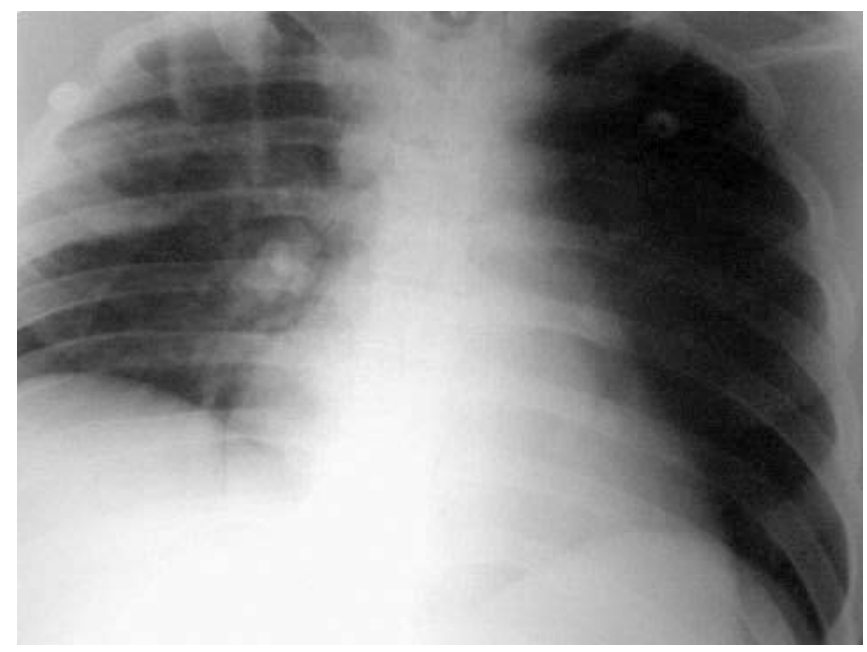

Figura 1 - Edema Agudo de Pulmão 


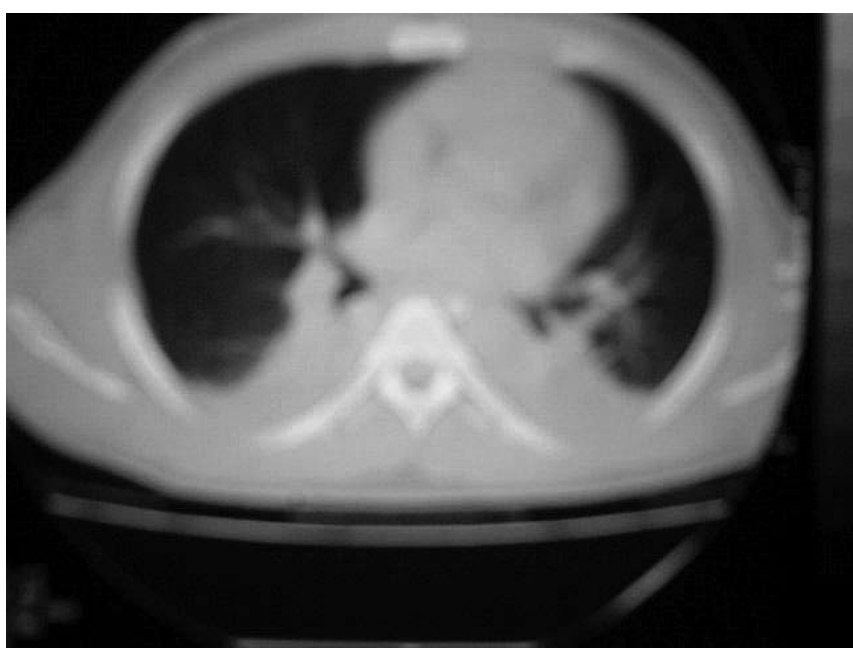

Figura 2 - Derrame Pleural

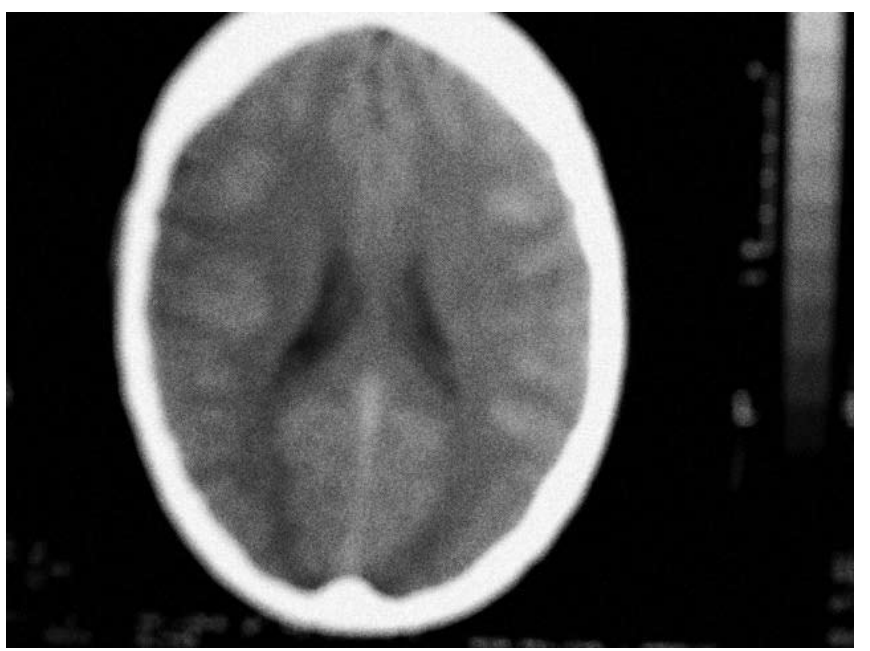

Figura 3 - Swelling Encefálico

\section{DISCUSSÃO}

Este relato de intoxicação aguda e intensa por óxido nítrico, parece inédito em nosso país e que teve desfecho favorável a despeito da gravidade, provavelmente pela rapidez em que as manobras de reanimação foram aplicadas, assim como, terapêutica adequada administrada. Paciente encontrado em coma com escala de Glasgow 8 com edema de face e língua, hiperemia de toda região cervical com insuficiência respiratória com cornagem em ambiente fechado, onde foi detectado vazamento de nitrogênio que vem de máquina utilizada para resfriamento de pista de gelo.

Pela dificuldade para intubação orotraqueal em decorrência do edema de laringe, constatou-se que houve aspiração de conteúdo gástrico para o sistema respi- ratório. Estes são sintomas clássicos de intoxicação pelo NO que causa edema de mucosa assim como necrose celular tanto da via respiratória alta como de bronquíolos terminais e alvéolos levando a um quadro de insuficiência respiratória alta assim como possibilidade de edema agudo de pulmão ${ }^{1}$, quadro este que se instala em decorrência de capilarite com aumento da permeabilidade e inundação alveolar, alem do aumento da pressão de perfusão pulmonar por vasodilatação seletiva da circulação pulmonar².

O NO em grande quantidade no ar ambiente compete com o oxigênio, levando a um quadro de hipóxia nas pessoas que a estão inalando, a ponto de terem quadro neurológico semelhante à encefalopatia difusa isquêmica, o que explicaria o coma deste paciente assim como os sinais de swelling encefálico detectados pela tomografia crânio ${ }^{3-5}$.

Seguramente houve uma associação de fatores que levaram a anóxia tanto ambientais como do próprio paciente (insuficiência respiratória alta e edema agudo de pulmão)

O tratamento instituído com ventilação mecânica associada à pressão expiratória positiva ajustada ao quadro respiratório, antibioticoterapia e corticoterapia foi suficiente para reverter o quadro de síndrome da insuficiência respiratória aguda (SARA) que se instalou neste caso ${ }^{6,7}$. O contato com o paciente foi rompido e a sua evolução a médio e longo prazo sob o ponto de vista do sistema respiratório, que seria importante inclusive na monitorização com testes ventilatórios para detectar possíveis complicações tardias de lesão pulmonar.

\section{REFERÊNCIAS}

01. Karlson-Stiber C, Hojer J, Sjoholm A et al - Nitrogen dioxide pneumonitis in ice hockey players. J Intern Med, 1996;239:451-456.

02. Kleinerman $\mathrm{J}$ - Some effects of nitrogen dioxide on the lung. Fed Proc, 1977;36:1714-1718.

03. Januszkiewicz AJ, Mayorga MA - Nitrogen dioxide-induced acute lung injury in sheep Toxicology. 1994;89:279-300.

04. Pelham TW, Holt LE, Moss MA - Exposure to carbon monoxide and nitrogen dioxide in enclosed ice arenas, Occup Environ Med, 2002;59:224233

05. Hedberg $\mathrm{K}$, Hedberg $\mathrm{CW}$, Iber $\mathrm{C}$ et al - An outbreak of nitrogen dioxide-induced respiratory illness among ice hockey players. JAMA, 1989;262:3014-3017.

06. Rosenlund M, Bluhm G - Health effects resulting from nitrogen dioxide exposure in an indoor ice arena. Arch Environ Health, 1999;54:52-57.

07. MorganWK- 'Zamboni disease'. Pulmonary edema in an ice hockey player. Arch Intern Med, 1995;155:2479-2480.

08. Persinger RL, Poynter ME, Ckless K et al - Molecular mechanisms of nitrogen dioxide induced epithelial injury in the lung. Mol Cell Biochem, 2002;234-235:71-80. 